\title{
The Potential Protective Effect of Mesencephalic Astrocyte-Derived Neurotrophic Factor on Post-Operative Delirium via Inhibiting Inflammation and Microglia Activation
}

Jing Liu' ${ }^{1} *$

Qiling Shen ${ }^{2, *}$

Huiping Zhang ${ }^{1} *$

Xueying Xiao'

Changming $\mathrm{Iv}^{3,4}$

Yueyue $\mathrm{Chu}^{2}$

Yujun Shen ${ }^{5}$

Dong Wang ${ }^{6}$

Qiying Shen $\mathbb{1 D}^{3,4}$

'Department of Anesthesiology, The Fourth Affiliated Hospital of Anhui

Medical University, Hefei, 230032,

People's Republic of China; ${ }^{2}$ Department of Bone Disease and Bone Tumor, The

First Affiliated Hospital of Anhui Medical University, Hefei, Anhui, 230022, People's

Republic of China; ${ }^{3}$ Department of

Anesthesiology, The First Affiliated Hospital of Anhui Medical University, Hefei, 230032, People's Republic of

China; ${ }^{4}$ Key Laboratory of

Anesthesiology and Perioperative

Medicine of Anhui Higher Education

Institutes, Anhui Medical University,

Hefei, Anhui, 230032, People's Republic

of China; ${ }^{5}$ Biopharmaceutical Research

Institute, Anhui Medical University, Hefei,

230032, People's Republic of China;

${ }^{6}$ School of Basic Medical Sciences, Anhui

Medical University, Hefei, 230032, Anhui,

People's Republic of China

*These authors contributed equally to this work

Correspondence: Qiying Shen; Dong Wang

Tel +86-055I-62922057

Email shenqiying-yy@I63.com;

wangdong@ahmu.edu.cn
Background: The increased inflammation is closely correlated with post-operative delirium (POD). Mesencephalic astrocyte-derived neurotrophic factor (MANF) shows protective effect on inflammatory diseases. However, the relationship between MANF and POD is still undefined. This study aimed to explore the potential effect of MANF on POD.

Methods: Pre- and post-operative levels of MANF and inflammatory cytokines were measured in serum from POD and non-POD patients by ELISA, as well as endogenous MANF in serum from healthy individuals with different ages. Endogenous MANF in mice brain from different ages was also measured. Abdominal surgery was performed for POD mice model. POD-like behavior changes in mice were evaluated using buried food test, open field test and Y maze test.

Results: Endogenous MANF was decreased in age-dependent manner in both humans and mice. The pre-operative level of MANF in serum from POD patients was lower compared with that in non-POD patients $(\mathrm{p}=0.016)$. MANF increase in serum after surgery was less in POD patients than that in non-POD patients $(\mathrm{p}<0.001)$. In mice, recombinant human MANF reversed the surgery-induced elongation of latency to eat food, increase in latency to center and increase in time in center in open field test, and also increase in duration in novel arm in $\mathrm{Y}$ maze test. In addition, MANF inhibited surgery-induced inflammation, microglial activation and M1 polarization in mice.

Conclusion: The relative low MANF level may contribute to POD in the elderly. MANF has a protective role against POD-like behavior changes in mice.

Keywords: MANF, post-operative delirium, inflammation, microglia

\section{Introduction}

Post-operative delirium (POD) is one of the common complications of the central nervous system after operation, which appears mainly in elderly patients. POD leads to poor prognosis, including increased length of hospital stay, hospitalization costs, functional disability, morbidity and even mortality. ${ }^{1}$ Although the underlying mechanism of POD is still unknown, more data have showed that the increased inflammatory response in circulation is positively correlated with the development of POD. ${ }^{2}$ Exploring the valuable indicators is able to facilitate the diagnosis and risk determination of POD, also shed lights on preventive measures or useful treatments for POD. ${ }^{3}$ Some biomarkers like the inflammatory IL-6 and IL-1 $\beta$ 
cytokines have been identified to contribute to the onset of POD in the elderly, but the mechanism is not clear. ${ }^{4,5}$

Inflammation, endoplasmic reticulum (ER) stress and oxidative stress have been reported to be involved in POD clinical manifestations. ${ }^{6,7}$ Mesencephalic astrocyte-derived neurotrophic factor (MANF) is an ER stress-inducible protein and plays a critical role in maintaining homeostasis. ${ }^{8,9}$ As a secretory protein, MANF was initially found as a neuroprotective factor. Recently, MANF has been reported to be expressed in multiple tissues and organs to show the positive effect on tissue damage repair and inflammatory inhibition. ${ }^{10-12}$ We have previously reported that MANF could directly interact with p65 and inhibit the expressions of Nuclear factor- $\kappa \mathrm{B}$ (NF- $\mathrm{\kappa B}$ )-dependent target genes. ${ }^{13}$ It is known that NF- $\mathrm{\kappa B}$ activation greatly promotes the expressions of pro-inflammatory cytokines, like IL-6, IL- $1 \beta$ and TNF- $\alpha$. The increased serum TNF- $\alpha$ and IL- 6 levels in POD patients have been demonstrated by the previous clinical study. ${ }^{14}$ Thus, MANF is possible to be involved in the onset of POD.
In this study, we aim to demonstrate the relationship between MANF and POD, especially explore whether MANF plays the protective effect against POD. We detected MANF level in patients' serum and mouse cortex to evaluate whether there was a correlation between MANF expression and POD onset. Also, we examined the effect of surgery on MANF expression in mouse brain. Moreover, the effect of recombinant human MANF protein on the onset of POD was explored.

\section{Materials and Methods}

A study overview of this research is established in Figure 1.

\section{Ethical Approval}

Ethical approval for the study was obtained from the First Affiliated Hospital of Anhui Medical University (PJ2017-0819) and registered at Chinese Clinical Trial Registry (ChiCTR1800017141). All the human individuals involved in this research had signed an informed consent, and the

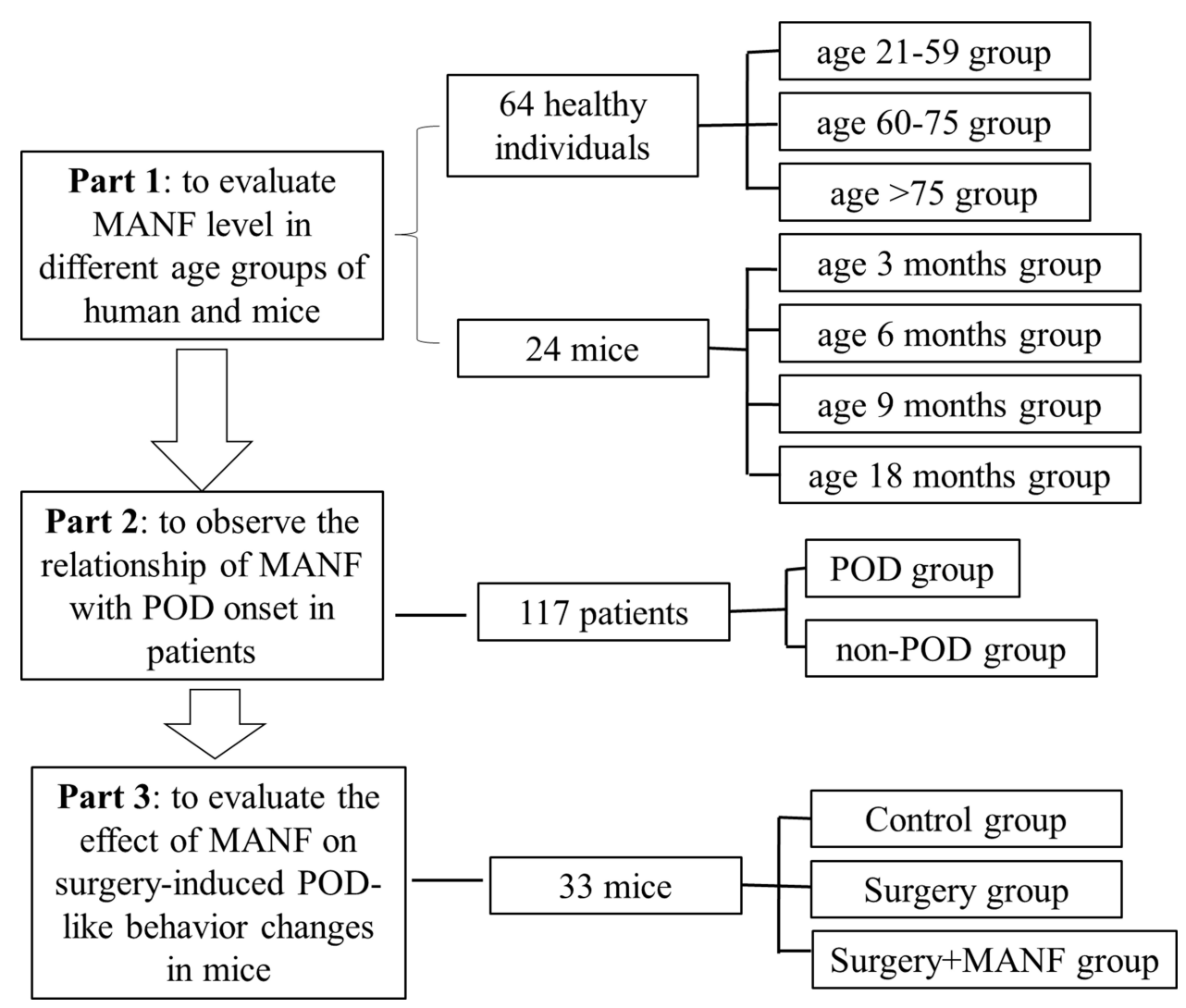

Figure I The study overview of this research. In this study, both human and mice were involved. To evaluate MANF level in different age groups of human and mice, 64 healthy individuals involved and 24 mice were used. To observe the relationship of MANF with POD onset, II7 patients were initially involved. To evaluate the effect of MANF on surgery-induced POD-like behavior changes in mice, 33 mice were used. 
study was conducted in accordance with the Declaration of Helsinki.

\section{Healthy Individual Enrollment and Blood Sample Collection}

For evaluation of MANF level in different age groups (age $21-59$, age $60-75$ or age $>75$ ), 64 healthy individuals were involved in this clinical study. None of these healthy individuals were diagnosed with diabetes or other endocrine diseases, which have been reported to make an effect on MANF expression. ${ }^{15,16}$ The traditional Confusion Assessment Method (CAM) and Mini-Mental State Examination (MMSE) were performed. Individuals with cognitive impairment (positive CAM results or MMSE score $<24$ ) were excluded. Blood samples were collected from these healthy individuals.

\section{Patient Enrollment, Blood Sample Collection, Delirium Assessment and Clinical Sample Size Definition}

117 patients undergo elective knee arthroplasty from September 2017 to December 2019 were initially involved in the clinical study. The traditional Confusion Assessment Method and Mini-Mental State Examination were performed among these patients before surgery. Patients with the positive CAM results and/or MMSE score $<24$ were diagnosed as pre-operative delirium and/or cognitive impairment, and were then excluded from the study. Also, CAM was used to assess POD on post-operative day 1 , day 2 and day 3, twice daily (in the morning and night shift) by specialized personnel. Due to preoperative cognitive dysfunction $(\mathrm{n}=8)$, declined to participation $(n=6)$ and other reasons $(n=34), 48$ patients were excluded from the clinical experiment and eventually 69 patients finished the whole study. According to the postoperative mental status assessment, 17 and 52 patients were diagnosed as POD (POD group, $n=17$ ) and nonPOD (non-POD group, $\mathrm{n}=52$ ), respectively. The basic information of these 69 involved patients is shown in
Table 1. The detailed procedure for patient enrollments and study of this part is illustrated in Supplemented Figure 1. Blood samples were collected from these patients at 24 hours before and after surgery.

The clinical sample size was calculated based on our pre-trial results of MANF levels in serum of patients in both POD and non-POD patients ( $\mathrm{n}=3$ in each group). In nonPOD group, the mean was 170 and the standard deviation was 30. In POD group, the mean was 143 and the standard deviation was 35 . The result was that we need to study more than 15 subjects to reject the null hypothesis that the population means of the POD and non-POD groups are equal with probability $1-\alpha=0.95$ and power equal to 0.80 .

\section{Animals, Behavior Tests and Sample Collection}

The female C57BL/6 mice with specific pathogen-free were purchased from Beijing Vital River Laboratory Animal Technology Co. Ltd. All mice experiments were approved by the Animal Care and Use Committee of Anhui Medical University (AHMU.LLSC.20190466). The welfare and treatment of the laboratory animals were followed with National Institute of Health Guide for the Care and Use of Laboratory Animals. For MANF detection in mice serum, mice in different ages (age 3 months, age 6 months, age 9 months, age 18 months) were grouped and sacrificed for cortex tissue sample collection. Each group had 6 mice $(n=6)$. For the experimental POD mouse model research, 3 months old mice were used and randomly divided into three groups: Control group, Surgery (laparotomy under 3\% Sevoflurane) and Surgery+MANF group (laparotomy under $3 \%$ Sevoflurane combined with intraperitoneal injection of $10 \mu \mathrm{g}$ human recombinant MANF protein). Each group had 11 mice $(\mathrm{n}=11)$. All mice had multiple behavioral tests in sequential order of buried food test (BFT), open field test (OFT) and Y maze test (YMT) at 24 hours before and after surgery respectively. The detailed methods were in accordance with the previous study ${ }^{17}$ and can be found in Supplemented Materials. At 24 hours after surgery, all mice were sacrificed to collect blood samples and brain tissues.

Table I Baseline Characteristics of These Patients Undergo Elective Knee Arthroplasty and Involved for MANF Detection

\begin{tabular}{|c|c|c|c|c|c|}
\hline Groups & Age (Years) & Gender (Male/Female) & BMI $\left(\mathrm{kg} / \mathrm{m}^{2}\right)$ & Diabetes (Yes/No) & ASA Grade (II/III) \\
\hline Non-PO $(n=52)$ & $73.29 \pm 6.96$ & $24 / 28$ & $23.68 \pm 3.71$ & $7 / 45$ & $12 / 42$ \\
\hline POD $(n=\mid 7)$ & $75.24 \pm 6.84$ & $10 / 7$ & $22.67 \pm 2.97$ & $2 / 15$ & $4 / 13$ \\
\hline$t / \chi^{2}$ & 1.005 & 0.823 & 1.017 & 0.000 & 0.000 \\
\hline$P$ & 0.318 & 0.364 & 0.313 & 1.000 & 1.000 \\
\hline
\end{tabular}




\section{ELISA Assay}

MANF, TNF- $\alpha$, IL- $1 \beta$ and IL- 6 levels in serum and brain tissues were detected by ELISA (MANF, Cloud-clone Crop, Wuhan, China; TNF- $\alpha /$ IL-1ß/IL-6, Cusabio, Wuhan, China). The procedure was performed in accordance with the protocols of ELISA kits.

\section{Western Blot Analysis}

MANF protein levels in brain tissues were detected by Western blot. Briefly, anti-MANF antibody (Abcam, MA, USA) and anti- $\beta$-actin antibody were used as primary antibodies. The anti-rabbit horseradish peroxidase (HRP)conjugated IgG was used as the secondary antibody. The results were visualized using enhanced chemiluminescence (Thermo Scientific, Tewksbury, MA, USA).

\section{Immunohistochemistry}

Paraffin-embedded brain tissue slides were immunestained to detect Iba-1 and iNOS. Detailed procedures were as the methods described in our previous research. ${ }^{18}$ Primary antibodies used here included Iba-1 (Abcam, MA, USA) and iNOS (Abcam, MA, USA). All sections were analyzed under a bright-field microscope.

\section{Statistical Processing}

We performed statistical analysis using SPSS 23.0. Measurement data were reported as mean value \pm error of the mean (SEM). Two samples $t$-test was used to compare data between two groups and one-way ANOVA was conducted to compare data among more than two groups. After ANOVA was done, Bonferroni comparison was used for post-hoc analysis to compare differences between groups. Qualitative data were expressed with relative number, Chi-square test was used to compare the difference between two groups, $P$ value $\leq 0.05$ was considered as of statistical significance.

\section{Results}

\section{MANF in Circulation and Cortex Was Decreased in an Age-Dependent Manner}

Firstly, we examined whether there was a difference of MANF level in circulation and cortex among the different age groups. Results from ELISA showed that MANF level in serum was decreased with aging (Figure 2A). POD-like behavior changes have been confirmed to be correlated with increased inflammatory response and neurodysfunction in pre-frontal cortex in animal studies. ${ }^{19,20}$ Results from Western blot showed that MANF reduced with aging in adult normal mice. MANF level in cortex of six-month, nine-month- and eighteen-month-old mice were significantly lower than that in three-month mice (Figure 2B). Therefore, the increased age is closely correlated with the decreased MANF expression in circulation and cortex, indicating the potential correlation of the reduced MANF level with POD among the aged patients.

\section{Postoperative MANF Increase Was Attenuated in POD}

Next, whether there was a relationship between MANF expression and POD occurrence was studied. We measured the pre-operative serum levels of MANF, TNF- $\alpha$, IL-1 $\beta$ and IL- 6 by ELISA, which showed that the pre-operative MANF level in POD patients was significantly lower than that in non-POD patients (Figure 3A). But, the pre-operative serum levels of TNF- $\alpha$, IL-1 $\beta$ and IL-6 in POD patients did not show significant differences compared with non-POD patients (Figure 3B-D). Under normal circumstances,
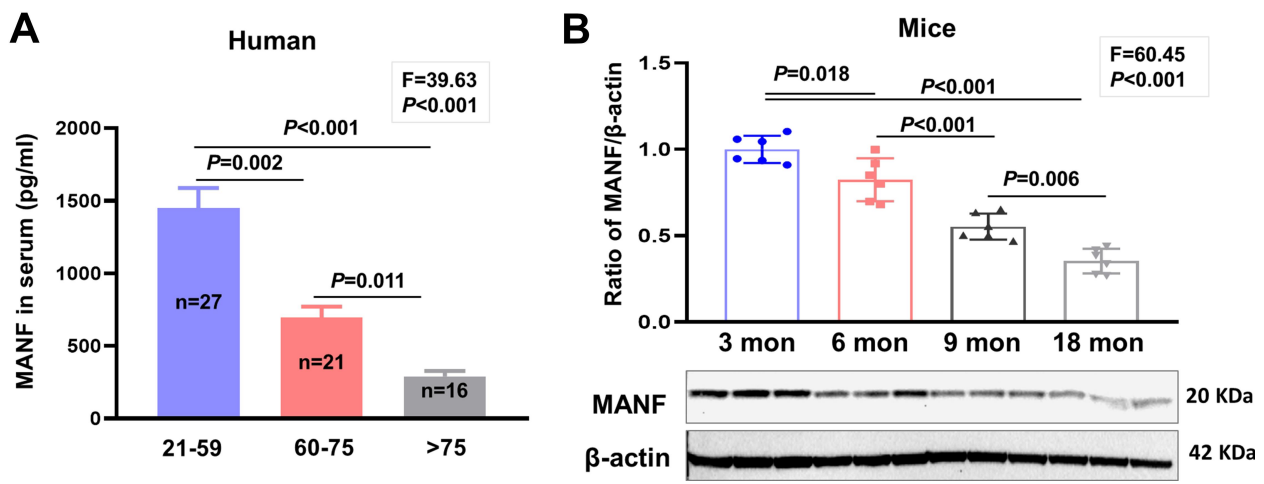

Figure 2 MANF decreased in an age-dependent manner in circulation and cortex. (A)The protein levels of MANF in human serum from healthy individuals in different age groups were measured by ELISA. (B)The endogenous protein levels of MANF in cortex of mice from different age groups were determined by Western blot analysis. Data are presented as the mean \pm SEM, $n=6$ per group for mice. 

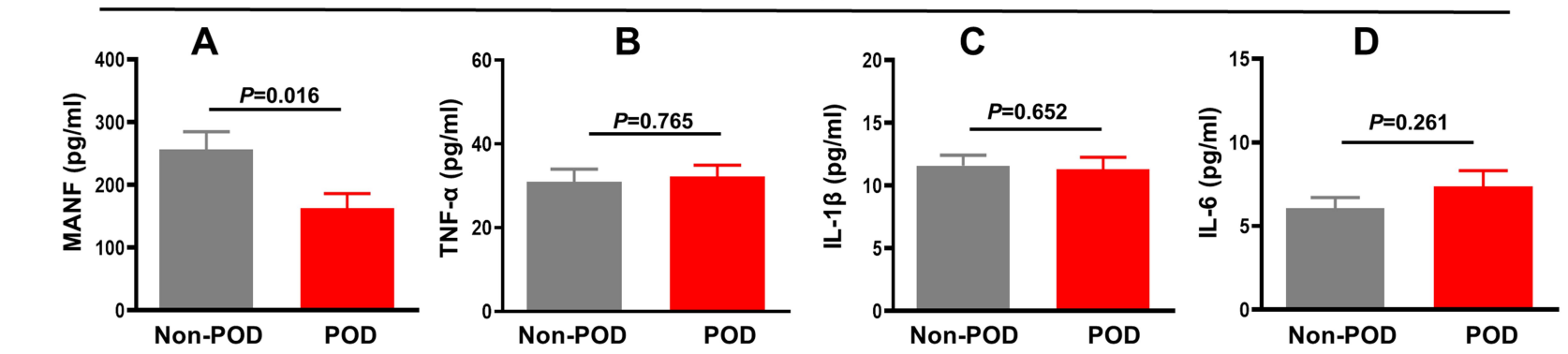

$P<0.001$

Variation of cytokines in serum (post-operative subtract pre-operative)
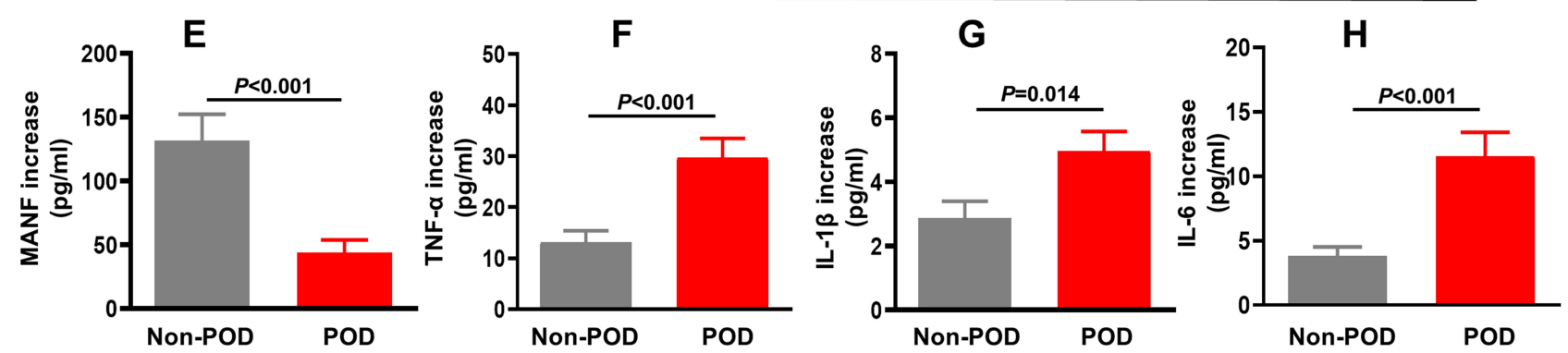

Figure 3 Relative lower baseline and less surgery-induced increase of endogenous MANF in POD patients than those in non-POD patients. ELISA kits were used to detect the pre-operative protein level of (A) MANF, (B) TNF- $\alpha$, (C) IL-I $\beta$ and (D) IL-6, in serum. Data are presented as the mean $\pm S E M$, Post-operative protein levels of MANF, TNF- $\alpha$, IL-I $\beta$, and IL- 6 in serum were detected to calculate the variations of these indicators after surgery $(\mathbf{E}-\mathbf{H})$.

surgical trauma is bound to cause stress response in the body and may have an effect on MANF protein level. Also, we detected MANF and inflammatory cytokine levels in circulation at 24 hours after surgery and made a comparison between POD and non-POD patients. Results showed that MANF was increased at 24 hours after surgery in both POD and non-POD groups (Figure 3E). The increase of MANF in the POD group was significantly lower compared with that in the non-POD group (Figure 3E). The serum levels of TNF- $\alpha$, IL-1 $\beta$ and IL-6 were increased after surgery as well. Similarly, POD patients had the greater increase of TNF- $\alpha$, IL-1 $\beta$ and IL-6 in serum compared with non-POD patients, which was in negative correlation with MANF variation after surgery (Figure $3 \mathrm{~F}-\mathrm{H}$ ). Therefore, compared with non-POD patients, the lower MANF level may contribute to more severe inflammation in POD patients after surgery.

\section{Endogenous MANF Was Up-Regulated in Mice Brain After Surgery}

Given that MANF was increased in circulation after surgery, we further asked whether surgery had the same effect on MANF level in brain. Figure 4A and B showed that MANF expression in prefrontal cortex was significantly increased in mice at 24 hours after surgery. These data suggest that surgery is able to enhance MANF protein level in brain tissues, which may affect the development of POD.

\section{Recombinant Human MANF Reversed Postoperative Delirium-Like Behavior Changes in Mice}

In order to explore the pathophysiological role of MANF increase after surgery and its effect on POD, we next evaluated the effect of recombinant MANF protein on POD-like behavior changes in mice. The detailed methods for behavior tests are illustrated in Figure 5A. Buried food test and open field test were used to assess natural behavior. Y maze test was used to assess learned behavior. As shown in Figure 5, MANF had the significant effect on some behavior changes induced by surgery. In buried food test, MANF reversed the surgery-induced elongation of latency of mice to eat food (Figure 5B). In open field test, MANF had no effect on freezing time, but significantly decreased surgery-induced increase in mice latency to center and increased the time of mice spent in center (Figure 5C-E). In $\mathrm{Y}$ maze test, MANF significantly increased the duration of mice in novel arm that was decreased by surgery (Figure 5F), but had no effect on entries to novel arm (Figure 5G). Both the total distance traveled by mice in open field test and the total number of 
A

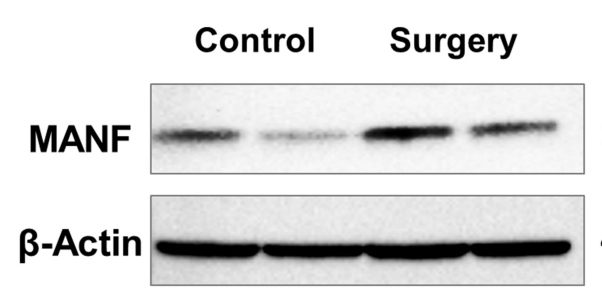

B

$20 \mathrm{KDa}$

$42 \mathrm{KDa}$

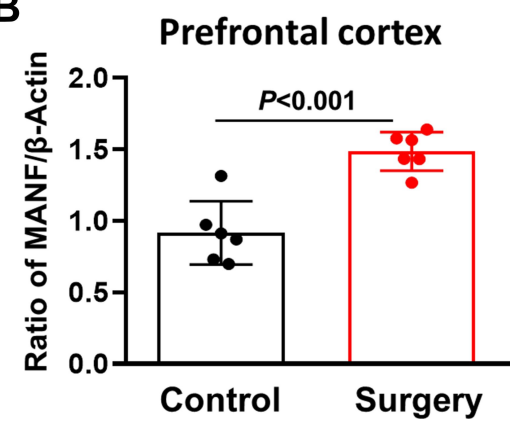

Figure 4 Endogenous MANF was up-regulated in mice brain 24 hours after surgery. (A) The protein levels of MANF in pre-cortex of mice from control group and surgery group.(B) The quantitative data of panel $A$. Data are presented as the mean $\pm S E M, n=6$ per group.
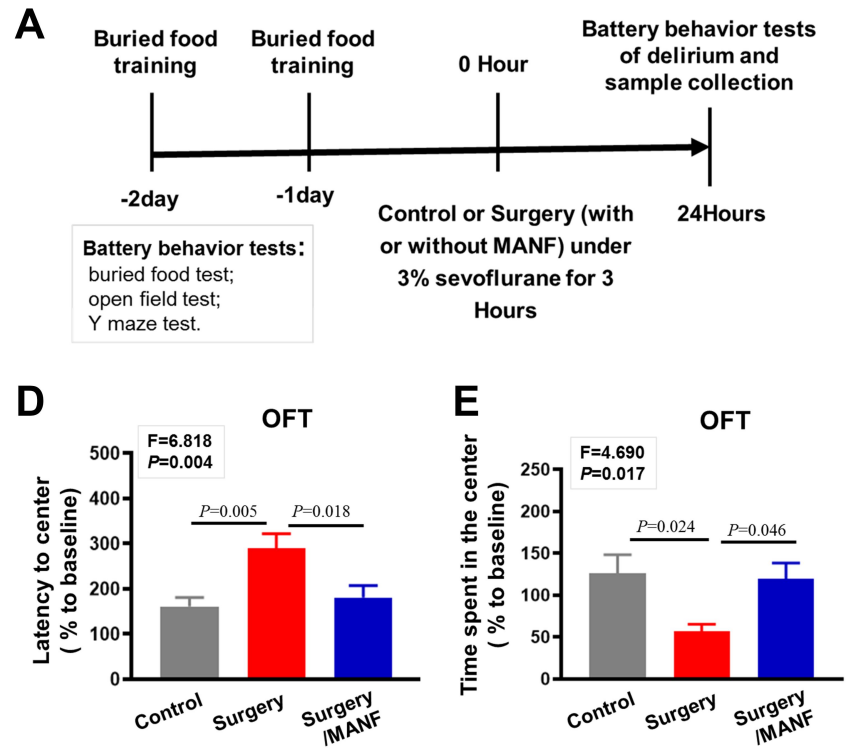
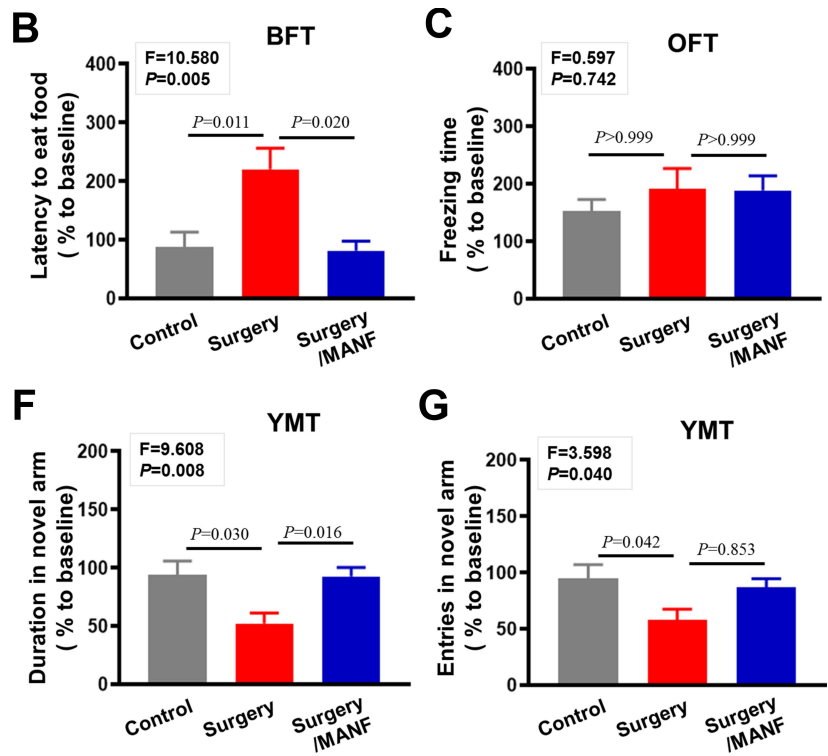

G

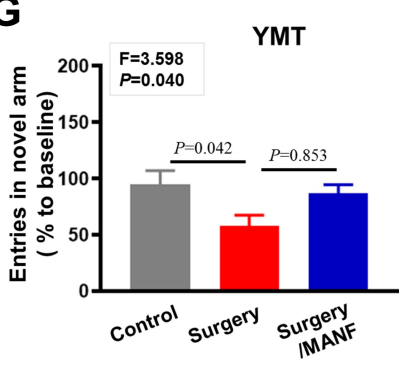

Figure 5 Recombinant human MANF protein reversed postoperative delirium-like behavior changes in mice. Multiple behavioral tests were performed to evaluated PODlike behavior changes in mice (A). The natural behaviors were assessed using buried food test (BFT) (B) and open field test (OFT) (C-E).The learned behaviors were assessed using $Y$ maze test (OFT) (F and $\mathbf{G}$ ). Data are presented as the mean \pm SEM, $n=I$ I per group.

arm visit of mice in Y maze test had no differences among the three groups (Supplemented Figure 2A and $\underline{B}$ ). These suggest that recombinant human MANF treatment is able to protect against the surgery-induced damage to natural and learned behaviors.

\section{Human Recombinant MANF Inhibited Surgery-Induced Inflammation, Microglial Activation and MI Polarization in Mice}

Several cytokines like TNF- $\alpha$, IL- 6 and IL-1 $\beta$ have been reported to contribute to neuroinflammation and are related to POD onset. ELISA results showed that MANF administration obviously decreased the surgery-induced up-regulation of
TNF- $\alpha$, IL-6 and IL-1 $\beta$ in serum (Figure $6 \mathrm{~A}-\mathrm{C}$ ). Besides, we also detected TNF- $\alpha$, IL-6 and IL- $1 \beta$ levels in prefrontal cortex. Human recombinant MANF treatment could reverse the surgery-induced increase of TNF- $\alpha$, IL-6 and IL-1 $\beta$ (Figure 6D-F). Therefore, the surgery-induced inflammation in mice is suppressed by recombinant human MANF protein. Also, we found that both the activated Iba- $1^{+}$microglia and iNOS $^{+} \mathrm{M} 1$ microglia were significantly increased in prefrontal cortex of mice with surgery compared with mice without surgery (Figure 7A-D), while these changes were reversed by recombinant human MANF treatment (Figure 7A-D). These results reveal that MANF is able to regulate microglia activation and polarization in mice after surgery, which contributes to the alleviation of brain inflammation. 


\section{Levels of cytokines in serum}
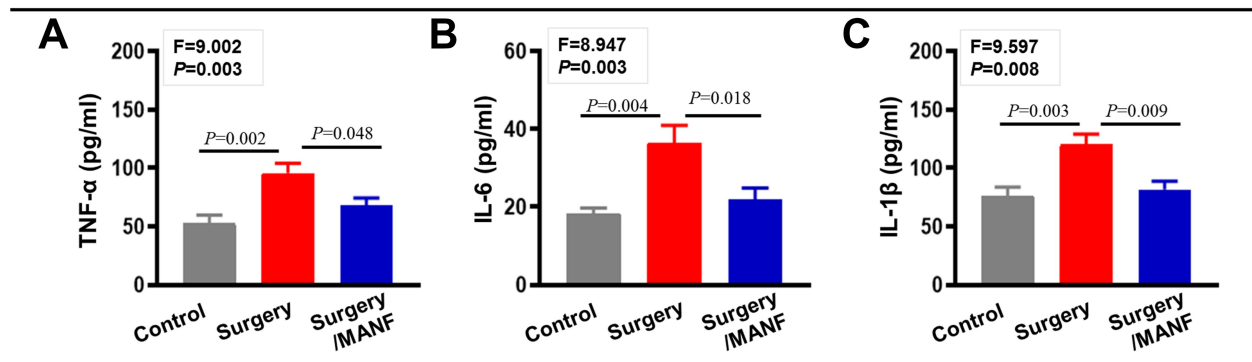

Protein levels of cytokines in prefrontal cortex
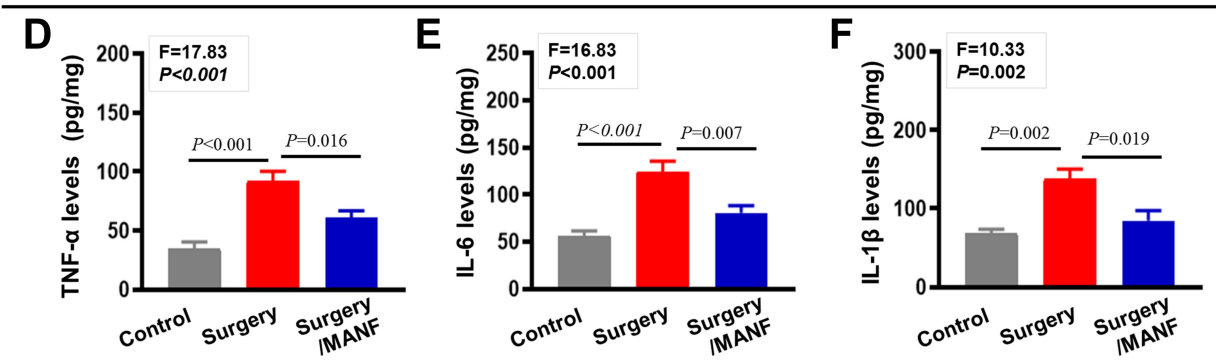

Figure 6 Recombinant human MANF protein inhibited surgery induced inflammatory response in mice. Levels of (A) TNF- $\alpha$, (B) IL-6, and (C) IL-I $\beta$ in serum were detected by ELISA assay. Protein levels of (D) TNF- $\alpha,(E)$ IL-6, and (F) IL-I $\beta$ in pre-cortex of mice were measured using ELISA kits. Data are presented as the mean \pm SEM, $n=6$ per group.
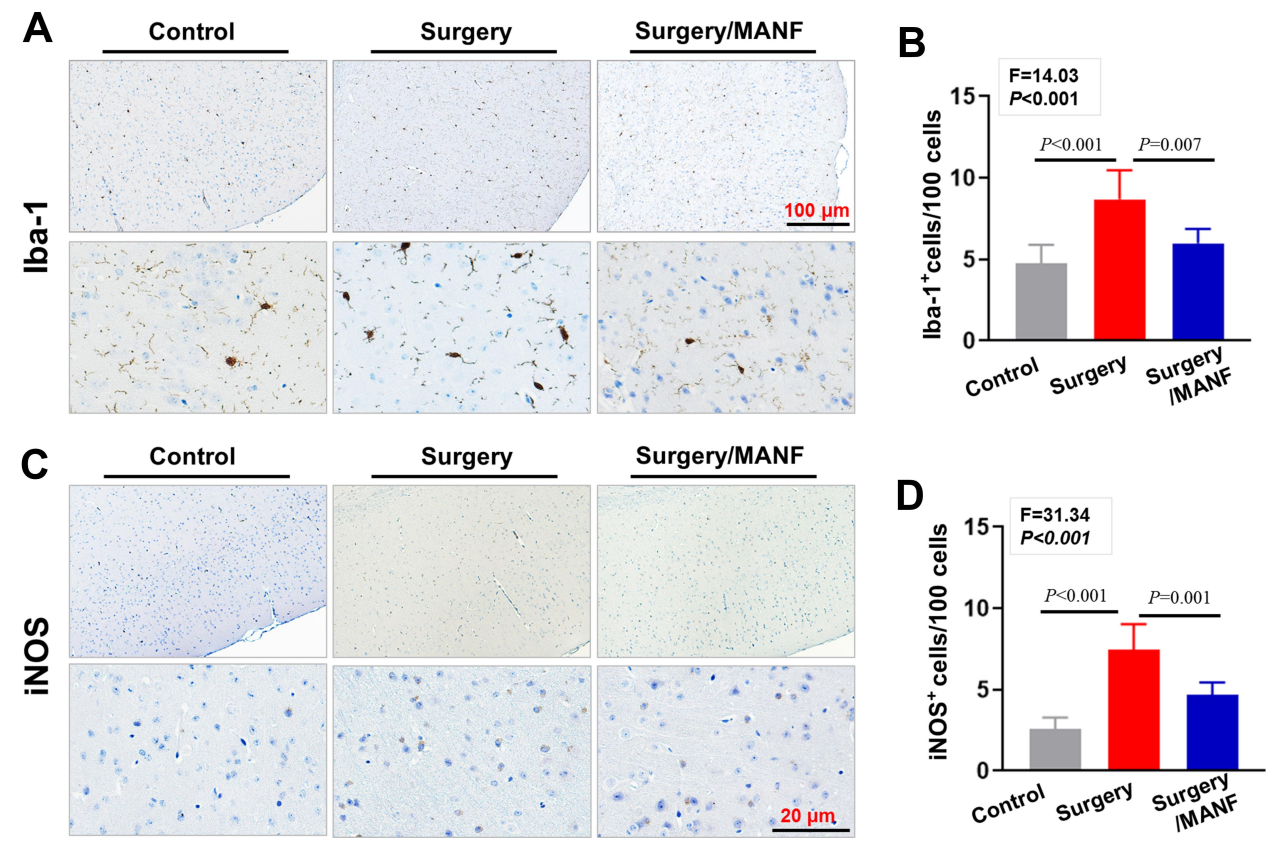

Figure 7 Recombinant human MANF inhibited surgery induced microglial activation and MI polarization. (A and B) Activated microglia cells in cortex of mice with or without surgery were detected by immunohistochemistry assay with specific antibodies of Iba-I and the number of Iba-I positive cells were calculated and compared between the three groups. (C and D) The brain tissues from control and surgery treated mice were performed by immunohistochemistry of iNOS and iNOS ${ }^{+}$cells were calculated. Data are presented as the mean $\pm \mathrm{SEM}, \mathrm{n}=6$ per group. 


\section{Discussion}

\section{The Effect of MANF Immunomodulation on POD}

As a neurotrophic factor, MANF has been demonstrated to be involved in many diseases of central nervous system and show the positive role in Parkinson's disease, acute brain injury and other neurodegenerative diseases. ${ }^{18} \mathrm{In}$ vitro, MANF shows neuroprotective effects on dopaminergic neurons and endogenous MANF is neuroprotective after Adeno-associated Virus (AAV) transfection. ${ }^{21,22}$ Matlik et al reported that administration of MANF promoted functional recovery of stroke-induced behavioral deficits in rat. Also, MANF could reduce the beta amylase-induced neuronal inflammatory response. ${ }^{9}$ However, the effect of MANF on POD is still undefined. Here, we firstly report the surgery-induced MANF increase in circulation and cortex, also firstly demonstrate the relatively low MANF level in POD patients. Furthermore, we evaluate the effect of human recombinant MANF protein on POD-like behavior changes in mice, which shows the protective role of human recombinant MANF in mice with surgery-induced POD-like behavior changes. The increase of pro-inflammatory factors like TNF- $\alpha$, IL-1 $\beta$ and IL- 6 is positively related to POD pathogenesis. ${ }^{23-26}$ The up-regulated inflammatory response has been also demonstrated in POD animal model studies. ${ }^{7,27,28}$ In this study, human recombinant MANF inhibited the surgeryinduced inflammatory response in mice to decrease the surgery-induced increase of IL-1 $\beta$, IL- 6 and TNF- $\alpha$ in serum and pre-cortex of mice brain, suggesting that MANF up-regulation in circulation and brain might be a self-protective response to surgery. Moreover, MANF modulated the surgery-induced microglia cells activation and M1 phenotype polarization. Because microglia cells play a pivotal role in initiating and enlarging neuroinflammation, it is possible that the positive effect of MANF on POD is mediated via its immune modulating function.

\section{The Relationship Between MANF Decrease and POD in the Elderly}

POD-like behavior changes are mainly related to neuroinflammation and the neural dysfunction. ${ }^{29}$ The decreased anti-inflammatory factors may worsen systemic inflammationassociated neurological damage. In this study, MANF inhibited the surgery-induced inflammatory response and protected mice against POD-like behavior impairment. For clinical samples, the increased TNF- $\alpha$, IL- $1 \beta$ and IL-6 in POD patients after surgery were more significant than that in non-POD patients. Therefore, we propose that the age-dependent decrease of MANF may be related to the high incidence of POD in the elderly. MANF decline may be related to the imbalance between anti-inflammatory and pro-inflammatory responses, as well as the high incidence of POD in elderly patients. The age-dependent MANF protein decline in mice cortex was found in this study, indicating the low MANF level might contribute to POD development.

The importance of systemic factors in the ageing process has been demonstrated in POD, but the underlying molecular mechanism is still unknown. The inflammation-induced occurrence of POD in the elderly is also multifactorial. Firstly, aging is an independent high-risk factor for POD. ${ }^{30,31}$ The imbalance of the pro- and anti-inflammatory systems may be a direct reason for the aging-caused excessive inflammation. Secondly, due to surgical trauma, the innate immune system is activated in a NF- $\kappa \beta$-dependent manner. ${ }^{29}$ Thirdly, alterations of endocrine function are considered as one reason for chronic inflammation in the aging process. ${ }^{32}$ Therefore, we propose that the low MANF level contributes to high incidence of POD by multiple ways: the low basic MANF level together with the declining ER stress-induced MANF secretion is not enough to effectively inhibit NF- $\mathrm{kB}$ activation; the low MANF level in brain cannot effectively restrict the initiation and enlarging of neuro-inflammation, finally leads to POD (see summary in Figure 8). For the mechanism on the low MANF expression in the elderly, it has been reported that there is the increased DNA methylation of BDNF (Brain Derived Neurotrophic Factor) and GDNF (Glial Cell Derived Neurotrophic Factor) genes in the elderly, ${ }^{33}$ which may lead to the decreased BDNF and GDNF expression. Therefore, we speculate that the declining MANF in the elderly is also possible to be attributed to the enhanced manf gene methylation. Also, BDNF and GDNF are the other two common neurotrophic factors playing key roles in the differentiation, growth and survival of neurons. Similar to MANF, BDNF and GDNF expressions have been reported to be decreased with age. ${ }^{33}$ But, whether the decreased BDNF and GDNF are associated with POD needs the further research.

\section{The Anti-Inflammatory Effect of MANF on POD}

Recently, researchers have focused on therapies and medicines effectively preventing and treating POD. ${ }^{34}$ Inflammation has been considered as an important target for prevention and treatment of POD. It has been reported that some drugs with 


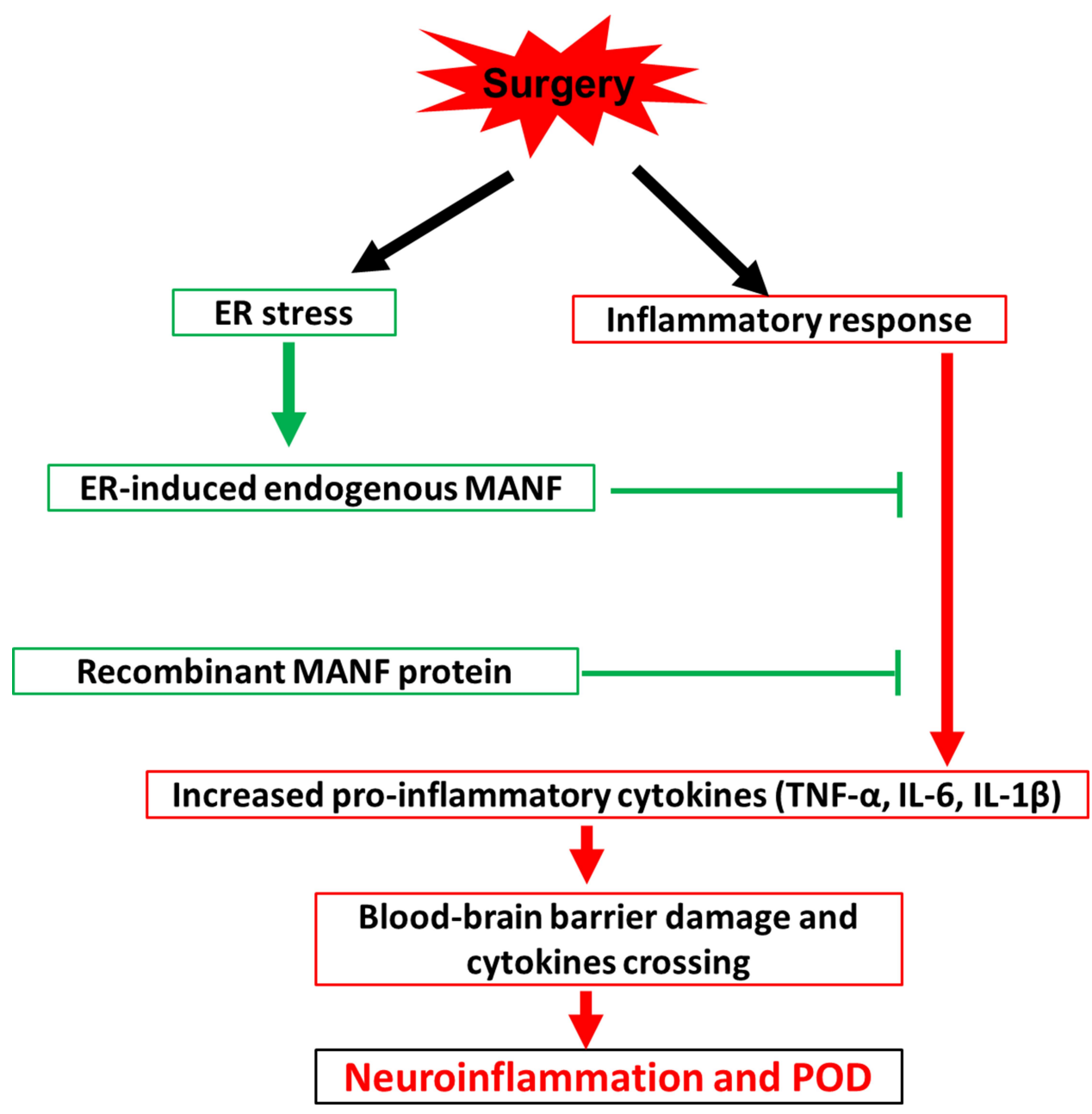

Figure 8 A summary of low level of MANF contributing to POD onset in the elderly.

neuroprotective effects are closely related to the antiinflammatory properties. ${ }^{35,36}$ However, the preventive role of these drugs for POD is still unclear, ${ }^{37}$ which may be attributed to the complex pathogenesis of POD and the undefined key regulatory molecules during POD development.

MANF has been reported to show the anti-inflammatory role in some inflammatory conditions. ${ }^{13}$ Both infection and sterile inflammation caused by various pathogenic factors will eventually activate the NF- $\kappa \mathrm{B}$ signal pathway and then result in the increased expressions of many proinflammatory cytokines including IL- 6 , IL- $1 \beta$ and TNF- $\alpha$. Here, we observed that the basic MANF level in the elderly with POD was decreased compared with that in non-POD ones. In contrast with the lower MANF level of POD patients, IL-6, IL- $1 \beta$ and TNF- $\alpha$ levels were increased significantly. The interaction of MANF with NF-кB p65 suggests that MANF may have a potential therapeutic effect to suppress the inflammatory response associated with surgical trauma and thus prevent POD development. In the future, we need more research to verify this hypothesis.

\section{Limitations}

There are still many deficiencies in this study. Firstly, due to medical ethical issues, it is very difficult for us to detect MANF protein level in the brain cortex of healthy adults with different ages. Secondly, this study is short of more in-depth mechanism exploration. But, these findings have already pointed out the direction for our future research on mechanism of POD and search for preventive strategies and effective treatments. Thirdly, POD mouse model used in this study could be improved by more quantifiable measurement of delirium-like phenotyping like electroencephalography (EEG). ${ }^{38}$ Taken together, our study indicates that MANF acts as a protective factor in POD by negatively regulating inflammatory response in circulation and pre-cortex via regulating activation and polarization of microglia cells (Figure 8). 


\section{Conclusion}

In this study, we found that MANF in both human circulation and mouse cortex was decreased in age-dependent manner. The serum MANF level was significantly lower in POD patients compared with that in non-POD patients. POD patients had the less serum MANF increase and greater pro-inflammatory cytokines increase after surgery compared with non-POD patients. Human recombinant MANF protein had the protective effect against POD in mice. Therefore, we demonstrate the protective role of MANF in POD via MANF-regulated microglia activation and neuroinflammation.

\section{Data Sharing Statement}

The data used to support the findings of this research are included with the article and are available from the corresponding author QiyingShen; shenqiying-yy@163.com.

\section{Acknowledgments}

We would like to thank Professor Yuxian Shen from the Anhui Medical University for technical assistance and thank Professor Yinguang Fan from Anhui Medical University for statistical assistant.

\section{Author Contributions}

All authors contributed to data analysis, drafting or revising the article, have agreed on the journal to which the article will be submitted, gave final approval of the version to be published, and agree to be accountable for all aspects of the work.

\section{Funding}

This work was supported by the funds from Natural Science Foundation of China to Qiying Shen and Dong Wang (no.81902003 and no.31800702), fund from University Natural Science Research Project of Anhui Province to Yujun Shen (KJ2018A0170).

\section{Disclosure}

The authors declare that they have no competing interests pertaining to this study.

\section{References}

1. Oh ST, Park JY. Postoperative delirium. Korean J Anesthesiol. 2019;72(1):4-12. doi:10.4097/kja.d.18.00073.1

2. Bendorius M, Po C, Muller S, Jeltsch-David H. From systemic inflammation to neuroinflammation: the case of neurolupus. Int $J$ Mol Sci. 2018;19(11):3588. doi:10.3390/ijms19113588
3. Liu X, Yu Y, Zhu S, Ma D. Inflammatory markers in postoperative delirium (POD) and cognitive dysfunction (POCD): a meta-analysis of observational studies. PLoS One. 2018;13(4):e0195659. doi:10.1371/journal.pone.0195659

4. Harris MJ, Brovman EY, Urman RD. Clinical predictors of postoperative delirium, functional status, and mortality in geriatric patients undergoing non-elective surgery for hip fracture. $J$ Clin Anesth. 2019;58:61-71. doi:10.1016/j.jclinane.2019.05.010

5. Casey CP, Lindroth H, Mohanty R, et al. Postoperative delirium is associated with increased plasma neurofilament light. Brain. 2020;143(1):47-54. doi:10.1093/brain/awz354

6. Wang Y, Shen X. Postoperative delirium in the elderly: the potential neuropathogenesis. Aging Clin Exp Res. 2018;30(11):1287-1295. doi:10.1007/s40520-018-1008-8

7. Zhang J, Gao J, Guo G, et al. Anesthesia and surgery induce delirium-like behavior in susceptible mice: the role of oxidative stress. Am J Transl Res. 2018;10(8):2435-2444.

8. Liu J, Zhou C, Tao X, et al. ER stress-inducible protein MANF selectively expresses in human spleen. Hum Immunol. 2015;76 (11):823-830. doi:10.1016/j.humimm.2015.09.043

9. Xu S, Di Z, He Y, et al. Mesencephalic astrocyte-derived neurotrophic factor (MANF) protects against $\mathrm{A} \beta$ toxicity via attenuating A $\beta$-induced endoplasmic reticulum stress. $J$ Neuroinflammation. 2019;16(1):35. doi:10.1186/s12974-019-1429-0

10. Danilova T, Belevich I, Li H, et al. MANF is required for the postnatal expansion and maintenance of pancreatic $\beta$-cell mass in mice. Diabetes. 2019;68(1):66-80. doi:10.2337/db17-1149

11. Liu J, Wu Z, Han D, et al. Mesencephalic astrocyte-derived neurotrophic factor inhibits liver cancer through Small Ubiquitin-Related Modifier (SUMO)ylation-related suppression of NF- $\mathrm{kB} /$ snail signaling pathway and epithelial-mesenchymal transition. Hepatology. 2020;71(4):1262-1278. doi:10.1002/hep.30917

12. Neves J, Zhu J, Sousa-Victor P, et al. Immune modulation by MANF promotes tissue repair and regenerative success in the retina. Science. 2016;353(6294):aaf3646. doi:10.1126/science.aaf3646

13. Chen L, Feng L, Wang X, et al. Mesencephalic astrocyte-derived neurotrophic factor is involved in inflammation by negatively regulating the NF-kB pathway. Sci Rep. 2015;5:8133. doi:10.1038/ srep08133

14. Zhang W, Wang T, Wang G, Yang M, Zhou Y, Yuan Y. Effects of dexmedetomidine on postoperative delirium and expression of IL-1 $\beta$, IL-6, and TNF- $\alpha$ in elderly patients after hip fracture operation. Front Pharmacol. 2020;11:678. doi:10.3389/fphar.2020.00678

15. Galli E, Härkönen T, Sainio MT, et al. Increased circulating concentrations of mesencephalic astrocyte-derived neurotrophic factor in children with type 1 diabetes. Sci Rep. 2016;6:29058. doi:10.1038/ srep29058

16. Wang $\mathrm{C}$, Yu S, Bao Q, et al. Circulating mesencephalic astrocyte-derived neurotrophic factor negatively correlates with atrial apoptosis in human chronic atrial fibrillation. J Cardiovasc Pharmacol. 2020;75(2):141-147. doi:10.1097/FJC.0000000000000781

17. Peng M, Zhang C, Dong Y, et al. Battery of behavioral tests in mice to study postoperative delirium. Sci Rep. 2016;6:29874. doi:10.1038/ srep29874

18. Yang W, Shen Y, Chen Y, et al. Mesencephalic astrocyte-derived neurotrophic factor prevents neuron loss via inhibiting ischemia-induced apoptosis. J Neurol Sci. 2014;344(1-2):129-138. doi:10.1016/j.jns.2014.06.042

19. Koyama T, Kawano T, Iwata $H$, et al. Acute postoperative pain exacerbates neuroinflammation and related delirium-like cognitive dysfunction in rats. J Anesth. 2019;33(3):482-486. doi:10.1007/ s00540-019-02635-3

20. Nishigaki A, Kawano T, Iwata H, et al. Acute and long-term effects of haloperidol on surgery-induced neuroinflammation and cognitive deficits in aged rats. J Anesth. 2019;33(3):416-425. doi:10.1007/ s00540-019-02646-0 
21. Mätlik K, Anttila JE, Kuan-Yin T, et al. Poststroke delivery of MANF promotes functional recovery in rats. Sci Adv. 2018;4(5):eaap8957. doi:10.1126/sciadv.aap8957

22. Hao F, Yang C, Chen SS, et al. Long-term protective effects of AAV9-mesencephalic astrocyte-derived neurotrophic factor gene transfer in parkinsonian rats. Exp Neurol. 2017;291:120-133. doi:10.1016/j.expneurol.2017.01.008

23. Yuan Y, Li Z, Yang N, et al. Exosome $\alpha$-synuclein release in plasma may be associated with postoperative delirium in hip fracture patients. Front Aging Neurosci. 2020;12:67. doi:10.3389/ fnagi.2020.00067

24. Çinar MA, Balikçi A, Sertoğlu E, Mehmet AK, Serdar MA, Özmenler KN. Role of CRP, TNF-a, and IGF-1 in delirium pathophysiology. Noro Psikiyatri Arsivi. 2014;51(4):376-382. doi:10.5152/npa.2014.6999

25. van Gool WA, van de Beek D, Eikelenboom P. Systemic infection and delirium: when cytokines and acetylcholine collide. Lancet 2010;375(9716):773-775. doi:10.1016/S0140-6736(09)61158-2

26. Mietani K, Sumitani M, Ogata T, et al. Dysfunction of the blood-brain barrier in postoperative delirium patients, referring to the axonal damage biomarker phosphorylated neurofilament heavy subunit. PLoS One. 2019;14(10):e0222721. doi:10.1371/journal. pone. 0222721

27. Skelly DT, Griffin ÉW, Murray CL, et al. Acute transient cognitive dysfunction and acute brain injury induced by systemic inflammation occur by dissociable IL-1-dependent mechanisms. Mol Psychiatry. 2019;24(10):1533-1548. doi:10.1038/s41380-019-0403-7

28. Plaschke K, Schulz S, Rullof R, Weigand MA, Kopitz J. In-depth characterization of the neuroinflammatory reaction induced by peripheral surgery in an animal model. J Neural Transmis. 2018;125 (10):1487-1494. doi:10.1007/s00702-018-1909-x

29. Bowman GL, Dayon L, Kirkland R, et al. Blood-brain barrier breakdown, neuroinflammation, and cognitive decline in older adults. Alzheimers Dement. 2018;14(12):1640-1650. doi:10.1016/j. jalz.2018.06.2857
30. Schenning KJ, Deiner SG. Postoperative delirium in the geriatric patient. Anesthesiol Clin. 2015;33(3):505-516. doi:10.1016/j. anclin.2015.05.007

31. Setters B, Solberg LM. Delirium. Prim Care. 2017;44(3):541-559. doi:10.1016/j.pop.2017.04.010

32. van den Beld AW, Kaufman JM, Zillikens MC, Lamberts SWJ, Egan JM, van der Lely AJ. The physiology of endocrine systems with ageing. Lancet Diabetes Endocrinol. 2018;6(8):647-658. doi:10.1016/S2213-8587(18)30026-3

33. Saito T, Braun PR, Daniel S, et al. The relationship between DNA methylation in neurotrophic genes and age as evidenced from three independent cohorts: differences by delirium status. Neurobiol Aging. 2020;94:227-235. doi:10.1016/j.neurobiolaging.2020.06.003

34. Kotekar N, Shenkar A, Nagaraj R. Postoperative cognitive dysfunction - current preventive strategies. Clin Interv Aging. 2018;13:2267-2273. doi:10.2147/CIA.S133896

35. Pluta MP, Dziech M, Czempik PF, Szczepańska AJ, Krzych ŁJ. Antipsychotic drugs in prevention of postoperative delirium-what is known in 2020? Int J Environ Res Public Health. 2020;17(17):6069. doi:10.3390/ijerph17176069

36. Xin X, Xin F, Chen X, et al. Hypertonic saline for prevention of delirium in geriatric patients who underwent hip surgery. $J$ Neuroinflammation. 2017;14(1):221. doi:10.1186/s12974-0170999-y

37. Scicutella A. The pharmacotherapeutic management of postoperative delirium: an expert update. Expert Opin Pharmacother. 2020;21 (8):905-916. doi:10.1080/14656566.2020.1738388

38. Au S, Roberts K, Cooler M, et al. Preoperative EEG: a potential predictor of postoperative delirium in older adults. J Am Geriatr Soc. 2021;69:S222-S223.
Journal of Inflammation Research

\section{Publish your work in this journal}

The Journal of Inflammation Research is an international, peerreviewed open-access journal that welcomes laboratory and clinical findings on the molecular basis, cell biology and pharmacology of inflammation including original research, reviews, symposium reports, hypothesis formation and commentaries on: acute/chronic inflammation; mediators of inflammation; cellular processes; molecular mechanisms; pharmacology and novel anti-inflammatory drugs; clinical conditions involving inflammation. The manuscript management system is completely online and includes a very quick and fair peerreview system. Visit http://www.dovepress.com/testimonials.php to read real quotes from published authors. 